in vivo $33: 85-91(2019)$

doi:10.21873/invivo.11443

\title{
Retinoic Receptor Signaling Regulates Hypertrophic Chondrocyte-specific Gene Expression
}

\author{
TSUYOSHI SHIMO ${ }^{1}$, EIKI KOYAMA ${ }^{2}$, TATSUO OKUI ${ }^{3}$, MASANORI MASUI ${ }^{3}$, \\ YUKI KUNISADA ${ }^{3}$, SOICHIRO IBARAGI ${ }^{3}$, NORIE YOSHIOKA ${ }^{3}$, NAITO KURIO ${ }^{4}$, \\ SHOKO YOSHIDA $^{3}$, AKIRA SASAKI ${ }^{3}$ and MASAHIRO IWAMOTO ${ }^{5}$ \\ ${ }^{1}$ Division of Reconstructive Surgery for Oral and Maxillofacial Region, \\ Department of Human Biology and Pathophysiology, School of Dentistry, \\ Health Sciences University of Hokkaido, Ishikari-Tobetsu, Japan; \\ ${ }^{2}$ Translational Research Program in Pediatric Orthopaedics, Division of Orthopaedic Surgery, \\ The Children's Hospital of Philadelphia, Philadelphia, PA, U.S.A.; \\ ${ }^{3}$ Department of Oral and Maxillofacial Surgery, Okayama University Graduate School of Medicine, \\ Dentistry, and Pharmaceutical Sciences, Okayama, Japan; \\ ${ }^{4}$ Department of Oral Surgery, Tokushima University Graduate School, Tokushima, Japan; \\ ${ }^{5}$ Department of Orthopaedics, University of Maryland School of Medicine, Baltimore, MD, U.S.A.
}

\begin{abstract}
Background/Aim: Retinoid signaling is important for the maturation of growth-plate chondrocytes. The effect of retinoid receptor gamma $(R A R \gamma)$ signaling on the expression of genes in hypertrophic chondrocytes is unclear. This study investigated the role of $R A R \gamma$ signaling in regulation of hypertrophic chondrocyte-specific genes. Materials and Methods: The gene expression in mouse E17.5 tibial cartilage was examined by in situ hybridization analysis. Real-time reverse transcription-polymerase chain reaction $(R T-P C R)$ and immunoblotting were used for analysis of $m R N A$ and phosphorylated mitogen-activated protein kinase (MAPK). Results: mRNA expression of Rarg and connective tissue growth factor (Ccn2) was detected in maturing chondrocytes throughout the cartilaginous skeletal elements. In chondrogenic ATDC5 cells, an RAR $\gamma$ agonist induced the gene expression of type-X collagen (Col10A1), transglutaminase-2 (Tg2), matrix metalloproteinase-13 (Mmp13), and Ccn2 mRNA, whereas a retinoic acid pan-agonist suppressed RAR $\gamma$ agonist-stimulated
\end{abstract}

This article is freely accessible online.

Correspondence to: Tsuyoshi Shimo, Division of Reconstructive Surgery for Oral and Maxillofacial Region, Department of Human Biology and Pathophysiology, School of Dentistry, Health Sciences University of Hokkaido, 1757 Ishikari-Tobetsu, Hokkaido 061-0293 Japan. Tel/Fax: +81 133231429, e-mail: shimotsu@hoku-iryou.ac.jp

Key Words: Retinoid signaling, matrix metalloproteinase, connective tissue growth factor, chondrocyte maturation, mitogenactivated protein kinase. gene expression. Phosphorylated extracellular signal regulated-kinases ( $p E R K 1 / 2), p-p 38$, and phosphorylated c-Jun $N$-terminal kinase ( $p J N K)$ MAPK were time-dependently increased by RAR $\gamma$ agonist treatment. Experimental p38 inhibition led to a severe drop in the RAR $\gamma$ agonist-stimulated expressions of CollOA1, Tg2, Mmp13, and Ccn2 mRNA. Conclusion: RAR $\gamma$ signaling is required for the differentiation of hypertrophic chondrocytes, with differential cooperation with $\mathrm{p} 38$ MAPK.

Skeletal growth is a tightly controlled process of growth plates that involves the proliferation and differentiation of chondrocytes (1) through the process of chondrocyte proliferation and the production of a matrix consisting mainly of type-II collagen and a large proteoglycan, aggrecan. When proliferative chondrocytes differentiate into hypertrophic chondrocytes, the extracellular matrix is replaced by type-X collagen (COL10A1).

Numerous studies have contributed to the characterization of the precise interplay of diverse factors that regulate hypertrophic chondrocyte differentiation, extracellular matrix (ECM) degradation, and bone formation and remodeling (27). Among these factors, cellular communication network factor 2 (CCN2), also known as connective tissue growth factor, is specifically expressed in hypertrophic chondrocytes, and is important for matrix remodeling, osteoclastogenesis, and angiogenesis in endochondral ossification (2, 3). Ccn2-knockout mice exhibited skeletal defects that included changes in chondrocyte proliferation and matrix gene expression (2). CCN2 seems to be a significant effector molecule in skeletogenesis, and appears 
to play particularly important roles in the terminal phases of chondrocyte maturation and endochondral ossification, phases that are also particularly sensitive to retinoid signaling (8). Several matrix metalloproteinases (MMPs), notably MMP13, are also crucial for endochondral ossification, catalyzing the degradation of collagen and aggrecan (5). Mice lacking MMP13 had severely impaired endochondral bone characterized by diminished ECM remodeling, prolonged chondrocyte survival, delayed vascular recruitment, and defective trabecular bone formation (6).

Retinoids are important regulators of the differentiation and cell proliferation in skeletal development, and they have been detected at higher concentrations in hypertrophic chondrocytes (9). Retinoids exert their effects by the modulation of gene expression by two distinct classes of nuclear receptors, the retinoic acid receptors $(\mathrm{RAR} \alpha,-\beta$ and $-\gamma)$ and the retinoid $\mathrm{X}$ receptors (RXR $\alpha,-\beta$ and $-\gamma)$, and an analysis of the effects of single and double RAR gene ablations in a murine model revealed compelling evidence that RARs are required for skeletal growth, matrix homeostasis, and growth plate function in postnatal mouse (10). RAR $\gamma$ has been demonstrated to be expressed specifically in the growth plate from the proliferative zone to the hypertrophic zone of chondrocytes (11). These and other studies have provided strong evidence that retinoids are involved in, and are required for, chondrocyte maturation. However, RAR $\gamma$ downstream effectors and the mechanisms of the signaling action in those processes remain largely unclear. The data that we obtained in the present study provide novel insights regarding the role of RAR $\gamma$ signaling in regulation of hypertrophic chondrocyte-specific genes.

\section{Materials and Methods}

In situ hybridization. Serial paraffin tissue sections of $\mathrm{C} 57 \mathrm{~B} 1 / 6$ mouse E17.5 tibial cartilage were pre-treated with $10 \mu \mathrm{g} / \mathrm{ml}$ proteinase K (Sigma) for $10 \mathrm{~min}$ at room temperature, post-fixed in $4 \%$ paraformaldehyde, washed with phosphate-buffered saline (PBS) containing $2 \mathrm{mg} / \mathrm{ml}$ glycine, and treated with $0.25 \%$ acetic anhydride in triethanolamine buffer (12). Sections were hybridized with antisense or sense ${ }^{35} \mathrm{~S}$-labeled probes (approximately $1 \times 10^{6}$ disintegrations/min (dpm)/section) at $50^{\circ} \mathrm{C}$ for $16 \mathrm{~h}$. Mouse cDNA clones included: Ccn2 (nt. 357-1180 and full length; NM_001901); matrix metalloproteinase-13 (Mmp13, nt. 11-744; NM_008607); collagen x (Col10A1) (nt. 1302-1816; NM009925); Rarg (nt. 16732628; NM_011244) and collagen II (Col2Al) (nt. 1095-1344; $\mathrm{X} 57982)$. After hybridization, slides were washed with $2 \times$ salinesodium citrate (SSC) containing $50 \%$ formamide at $50^{\circ} \mathrm{C}$, treated with $20 \mu \mathrm{g} / \mathrm{ml} \mathrm{RNase} \mathrm{A}$ for $30 \mathrm{~min}$ at $37^{\circ} \mathrm{C}$, and washed three times with $0.1 \times \mathrm{SSC}$ at $50^{\circ} \mathrm{C}$ for $10 \mathrm{~min} /$ wash. Sections were dehydrated with 70,90 , and $100 \%$ ethanol for $5 \mathrm{~min} / \mathrm{step}$, coated with Kodak NTB-3 emulsion (Carestream Health, Inc., Rochester, NY, USA) diluted 0.8:1 with water, and exposed for 10 to 14 days. Slides were developed with Kodak D-19 at $20^{\circ} \mathrm{C}$ and stained with hematoxylin. Dark and bright field images for radiolabeled riboprobes of each cDNA clone were captured using a digital camera.
Cell culture. A murine chondrogenic cell line, ATDC5, was purchased from the RIKEN Cell Bank (Tsukuba Science City, Japan). ATDC5 cells were cultured at a density of $1 \times 10^{4}$ cells $/ \mathrm{cm}^{2}$ in a 1:1 mixture of Dulbecco's modified Eagle's medium (DMEM) and Ham's F12 medium (GIBCO/BRL, Gaithersburg, MD, USA) containing 5\% fetal bovine serum (FBS; Hyclone, Logan, UT, USA), followed by replacement with DMEM/F12 containing 5\% FBS, $10 \mu \mathrm{g} / \mathrm{ml}$ human recombinant insulin (Wako Pure Chemical, Osaka, Japan), $10 \mu \mathrm{g} / \mathrm{ml}$ transferrin (Roche Diagnostics, Mannheim, Germany) and $3 \times 10^{-8} \mathrm{M}$ sodium selenite (Sigma) for the promotion of cell differentiation, and subsequent culture at $37^{\circ} \mathrm{C}$ for different periods up to 12 days under $5 \% \mathrm{CO}_{2}$. RNA was extracted from cultured cells when the ATDC 5 cells became confluent (4 days after plating) and was then extracted every 2 days after confluence.

Day-10 cultures of ATDC5 cells were treated with $100 \mathrm{nM}$ alltrans-retinoic acid (ATRA; Sigma St Louis, MO, USA); $100 \mathrm{nM}$ RAR $\gamma$-selective agonist AGN204647 (10), 100 nM RAR $\alpha$-selective agonist AGN195183 (13), the $100 \mathrm{nM}$ RAR inverse agonist AGN194310 (10), $20 \mu \mathrm{M}$ of selective inhibitor of extracellular signal regulated-kinases (ERK1/2) kinase PD98059 (Calbiochem, La Jolla, CA, USA), $20 \mu \mathrm{M}$ of selective inhibitor of p38 kinase SB203580 (Calbiochem), $20 \mu \mathrm{M}$ of selective inhibitor of c-Jun $N$ terminal kinase (JNK) SP60012 (Calbiochem), or combinations of these agents for $24 \mathrm{~h}$.

Immunoblot analysis. An immunoblot analysis for mitogen-activated protein kinase (MAPK) and activated MAPK by using cell lysates from the experimental cultures. The cells were lysed in an ice-cold lysis buffer (50 mM Tris-HCl, $\mathrm{pH} 7.4$, containing $150 \mathrm{mM} \mathrm{NaCl}, 1 \%$ Triton X-100, 1\% NP-40, $10 \mathrm{mM} \mathrm{NaF,} 100 \mathrm{mM}$ leupeptin, $2 \mathrm{mg} / \mathrm{ml}$ aprotinin, and $1 \mathrm{mM}$ phenylmethyl sulfonyl fluoride). The lysates were centrifuged at $16,000 \times \mathrm{g}$ for $20 \mathrm{~min}$ at $4^{\circ} \mathrm{C}$, and the protein concentrations in the supernatant were determined by a BCA assay. A 50- $\mu$ g sample of each lysate was subjected to $12 \%$ sodium dodecyl sulfate-polyacrylamide gel electrophoresis. The proteins were transferred to nylon membranes (Immobilon-P, Millipore, Bedford, MA, USA). The membrane was incubated with primary and secondary antibodies according to the ECL chemiluminescence protocol (RPN2109; Amersham Biosciences, Buckinghamshire, UK) to detect secondary antibody binding. Antibodies to ERK1/2, phosphorylated (p)ERK1/2, p38, p-p38, JNK, p-JNK, activating transcription factor 2 (ATF2), and p-ATF2 were purchased from Cell Signaling Technology (Danvers, MA, USA) and used at 1:200 dilution. Horseradish peroxidase-conjugated anti-mouse, rabbit, or goat immunoglobulins (IgGs) were used as the secondary antibodies at 1:1,000 dilution.

p38 MAPK assay. Cell extracts were incubated overnight with immobilized p38 MAPK (Thr180/Tyr182) monoclonal antibody (Cell Signaling Technology). A kinase reaction was performed in the presence of $100 \mu \mathrm{M}$ of cold ATP (Cell Signaling Technology) and $2 \mu \mathrm{g}$ of ATF2 fusion protein (Cell Signaling Technology). The phosphorylation of ATF2 at Thr71 was measured by western blotting using phospho-ATF2 (Thr71) antibody (Cell Signaling Technology).

Real-time reverse transcription-polymerase chain reaction (RT-PCR). Total RNA was isolated from ATDC5 cells by using TRIZOL reagent (Life Technologies, Rockville, MD, USA) according to the manufacturer's recommendations, and the transcripts were analyzed 

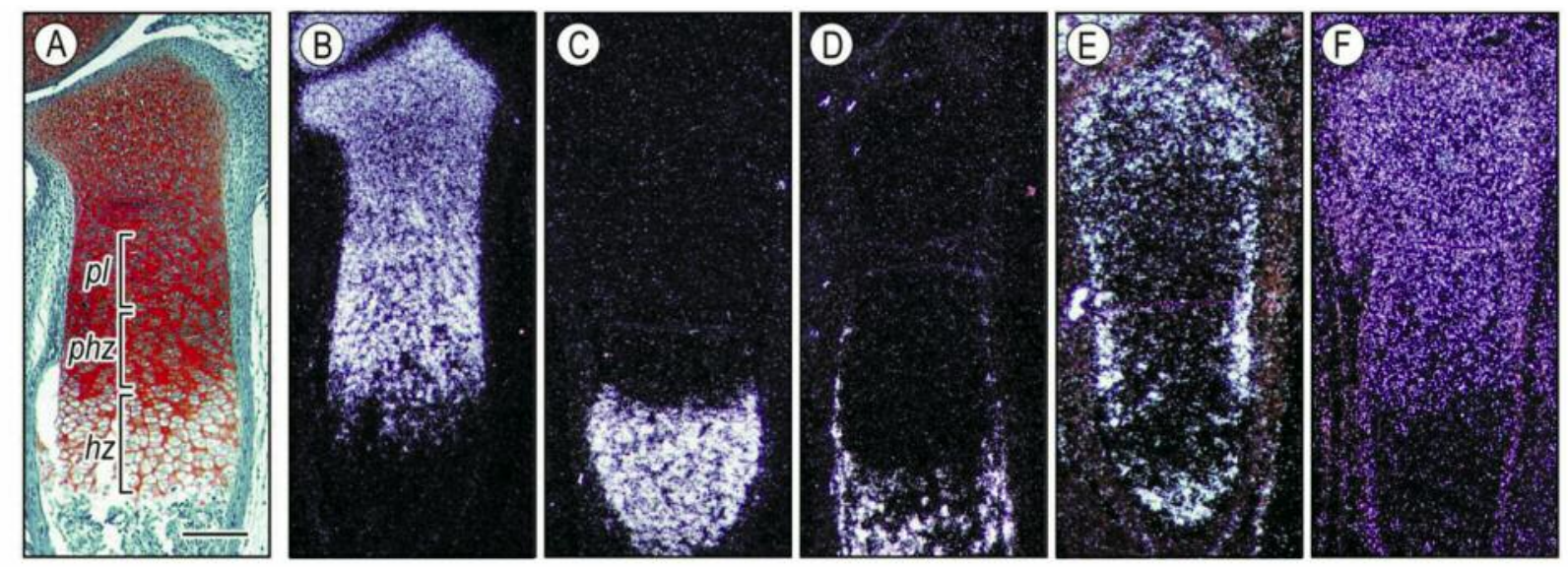

Figure 1. In situ hybridization analysis of gene expression in mouse E17.5 tibial cartilage. Longitudinal serial sections were processed for histochemical proteoglycan staining with safranin $O(A)$, in situ hybridization with 35S-UTP riboprobes encoding type-II collagen (B), type-X collagen $(C)$, matrix metalloproteinase-13 (D), connective tissue growth factor $(C c n 2)(E)$ and retinoid receptor gamma $(R a r g)(F)$. Note the strong expression of Rarg and Ccn2 in maturing chondrocytes and up-regulation of Ccn2 transcripts in hypertrophic chondrocytes in tibial growth plates. pl: Proliferating chondrocyte zone, phz: pre-hypertrophic chondrocyte zone, and hz: hypertrophic chondrocyte zone. Scale bar in A for A-F: 75 um.

by real-time RT-PCR. The primers used were the following: 5'TGAGAGAGGCGAATGGAACG-3' (forward) and 5'-TTCTGCC CGAGGGTTCTAGC-3' (reverse) for aggrecan (Agr); 5'-AAGAA CAGCATCGCCTACCT-3' (forward) and 5'-CTTACCAGTGT GTTTCGTGC-3' (reverse) for Col2; 5'-TCTGTACAATAGG CAGCAGC-3' (forward) and 5'-TAGGCGTGCCGTTCTTATAC-3' (reverse) for Col10A1; 5'-TTCCGAGTATGACT-3' (forward) and 5'GCCAATATCAGTCGGGAACA-3' (reverse) for TG-2; 5'-CCAGA CTATGGACAAAGATT-3' (forward) and 5'-ATGCGATTACTCCAG ATACT-3' (reverse) for Mmp 13; 5'-CCAATGACAATACCTTCTGC3' (forward) and 5'-GAAAGCTCAAACTTGACAGG-3' (reverse) for Ccn2; and 5'-TGAACGGGAAGCTCACTGG-3' (forward) and 5'TCCACCACCCTGTTGCTGTA-3' (reverse) for glyceraldehyde 3phosphate dehydrogenase Gapdh. The real-time RT-PCR was performed with a Light Cycler (Roche Molecular Biochemicals, Mannheim, Germany) in Light Cycler capillaries with a commercially available master mix containing Taq DNA polymerase and SYBRGreen I deoxyribonucleoside triphosphate (Light Cycler DNA Master SYBR-Green I; Roche Molecular Biochemicals). After the addition of primers (final concentration: $10 \mu \mathrm{M}), \mathrm{MgCl}_{2}(3 \mathrm{mM})$, and template DNA to the master mix, 65 cycles of denaturation $\left(95^{\circ} \mathrm{C}\right.$ for $\left.15 \mathrm{~s}\right)$ and extension $\left(60^{\circ} \mathrm{C}\right.$ for $\left.45 \mathrm{~s}\right)$ were performed. After the completion of PCR amplification, a melting curve analysis was conducted.

Statistical analysis. The data were analyzed using the unpaired Student's $t$-test for the comparisons of two groups and a one-way analysis of variance (ANOVA) for the analysis of repeated multiple group comparisons. Results are expressed as the mean \pm standard deviation (SD). $p$-Values of less than 0.05 were considered significant.

\section{Results}

Expression of Rarg and Ccn2 in developing tibial cartilage. In a first set of experiments, we determined the expression patterns of Rarg and Ccn2 in mouse developing cartilage.
Longitudinal serial sections of tibial cartilage from E17.5 mice were processed for in situ hybridization using 35Slabeled antisense riboprobes. Tibial growth plates exhibited distinctive morphological characteristics and chondrocyte organization. They displayed prospective articular chondrocytes at their epiphyseal ends and long growth plates with well-define proliferative, prehypertrophic and hypertrophic zones occupying the metaphysis and diaphysis (Figure 1A). In addition, the diaphysis underwent the process of endochondral ossification and was surrounded by intramembranous bony collar. In situ hybridization on serial sections of tibial cartilage revealed that while Col2 was strongly expressed throughout most chondrocytes except for hypertrophic chondrocytes, ColloAl was markedly upregulated in the hypertrophic chondrocytes (Figure 1B and C, respectively). Mmp13 transcripts were confined to the post-hypertrophic chondrocytes, and cells residing in the primary spongiosa and intramembranous bony collar (Figure 1D). Expression of Ccn2 and Rarg was detected in maturing chondrocytes throughout the cartilaginous skeletal elements, indicating a significant similarity between topographical distribution between the two molecules (Figure 1E and F, respectively). Importantly, $C c n 2$ expression became abundant in the hypertrophic zone of growth plate. These data clearly indicate that expression of Rarg and Ccn2 detected in maturing chondrocytes precedes chondrocyte hypertrophy characterized by CollOA1 and Mmpl3 expression.

$R A R \gamma$ agonist-dependent modulation of ColloA1, Tg2, Mmp13 and Ccn2 gene expression. Confluent ATDC5 cells were switched to low-serum-containing medium and treated 

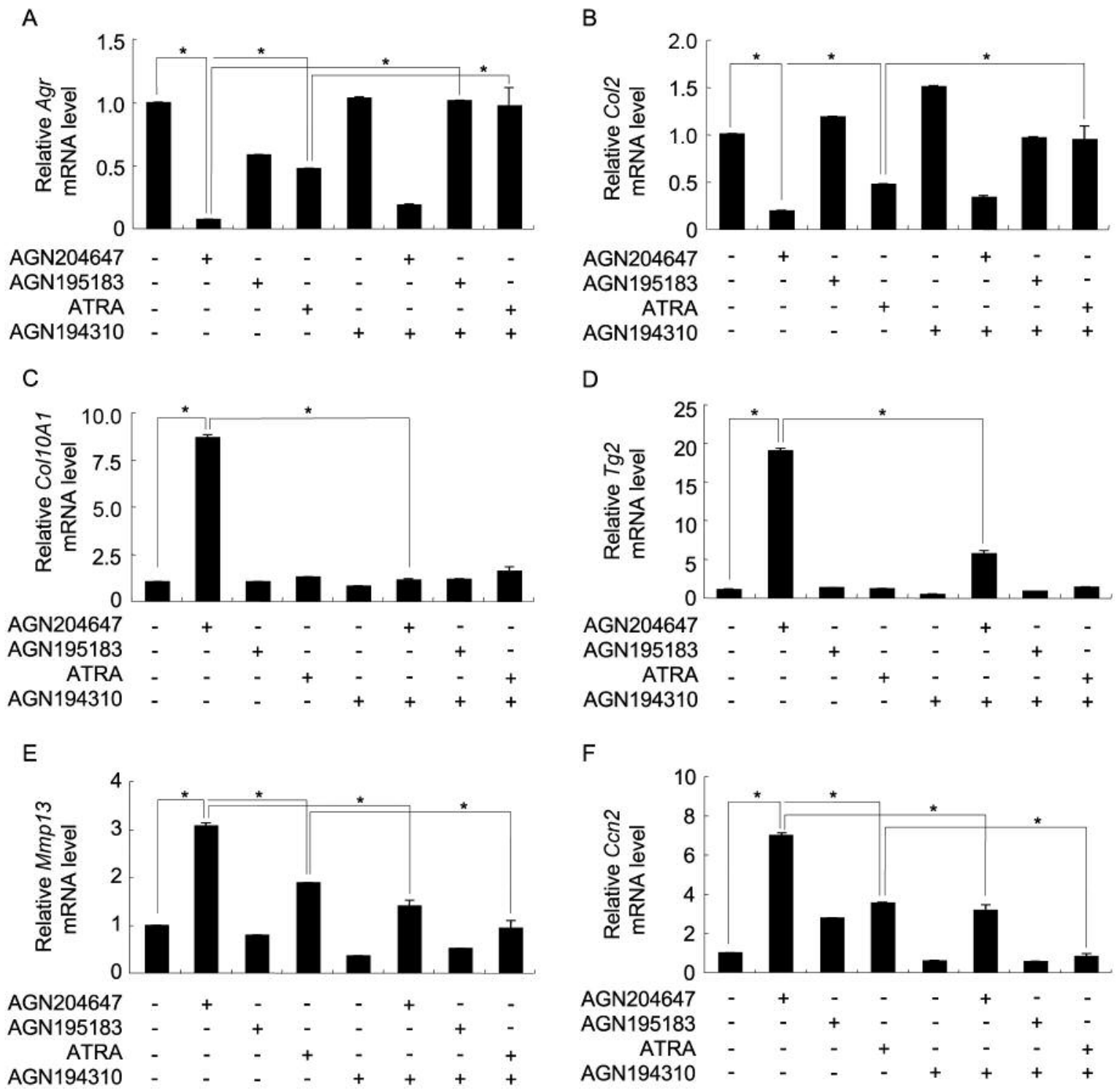

Figure 2. Modulation of aggrecan (Agr) (A), type II collagen (Col2) (B), type X collagen (Col10A1) (C), transglutaminase-2 (Tg2) (D), matrix metalloproteinase-13 (Mmp13) (E), and connective tissue growth factor (Ccn2) (F) mRNA expression by retinoid receptor (RAR)- $\alpha$ (AGN195183) or - $\gamma$ (AGN204647) agonists with/without retinoid pan-antagonist (AGN194310) in 10-day cultures of ATDC5 cells. ATDC5 cells were exposed to 100 nM AGN204647, AGN195183, or all-trans-retinoic acid (ATRA) with or without $100 \mathrm{nM}$ AGN194310 for $24 \mathrm{~h}$. Total RNA from these cells was used for real-time reverse transcription-polymerase chain reaction analysis. The values in the graph indicate the relative $m R N A$ level. *Significantly different at $p<0.05$ between the indicated groups.

with RAR $\gamma$ agonist AGN204647, RAR $\alpha$ agonist AGN195183, or ATRA for $24 \mathrm{~h}$ in the absence or presence of retinoid antagonist AGN194310 and were then subjected to real-time RT-PCR analysis. RAR $\gamma$ agonist significantly down-regulated aggrecan and Col2 mRNA, whereas retinoid antagonist reversed down-regulation of aggrecan and Col2 mRNA induced by RAR $\gamma$ agonist (Figure 2A and B). RAR $\gamma$ agonist significantly boosted the mRNA expressions of Mmpl3 and
Ccn2 compared with the control, whereas retinoid antagonist reversed RAR $\gamma$ agonist action as well as Col10Al and $T g 2$ mRNA expression (Figure 2C-F).

Involvement of MAPK. To verify that $\operatorname{RAR} \gamma$ agonist treatment modulated the activation of MAPK, cultures were treated with AGN204647 for different lengths of time and the cultures were processed for an immunoblot analysis of 
A

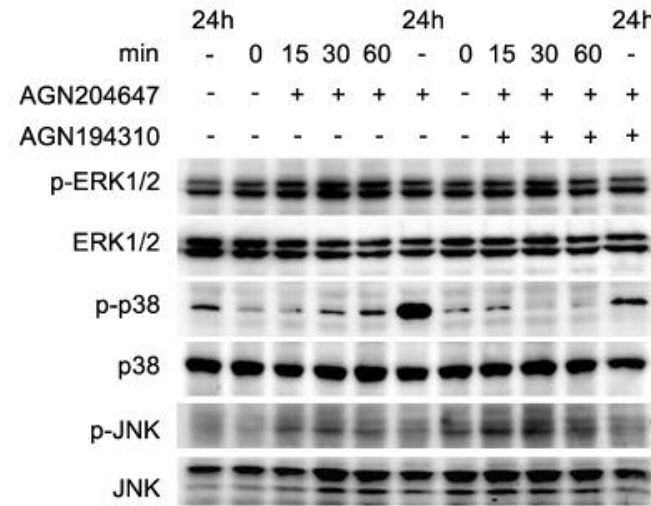

D

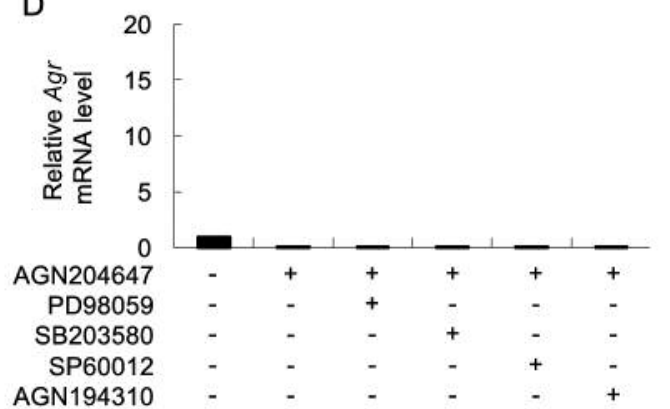

$\mathrm{F}$
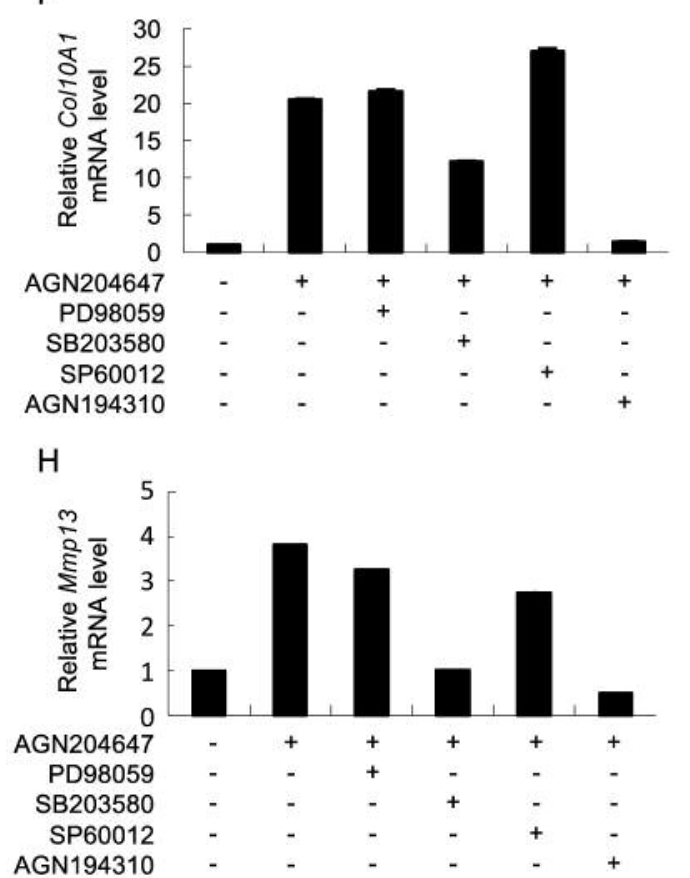

B

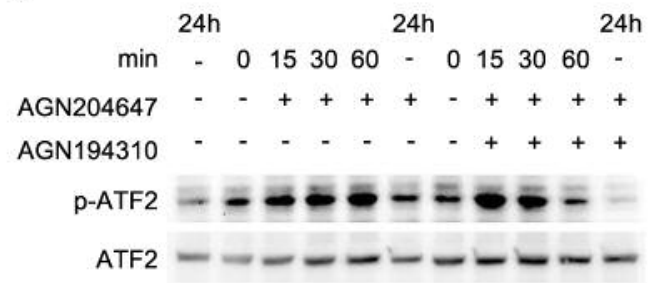

C
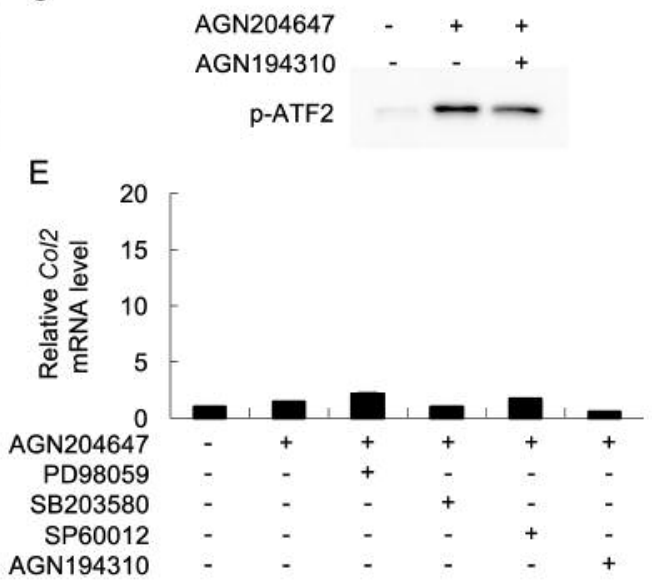

G
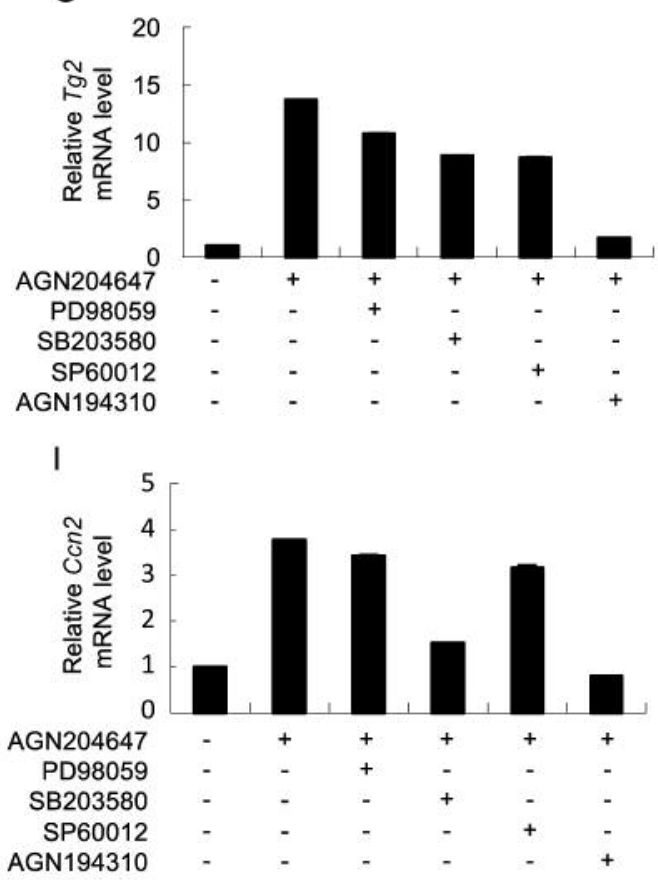

Figure 3. Analysis of the action of mitogen-activated protein kinase (MAPK) on aggrecan (Agr), type II collagen (Col2), type X collagen (ColloA1), transglutaminase-2 (Tg2), matrix metalloproteinase-13 (Mmp13), and connective tissue growth factor (Ccn2) mRNA induction by retinoid receptor gamma $(R A R \gamma)$ agonist AGN204647. ATDC5 cells were exposed to $100 \mathrm{nM}$ AGN204647 with/without $100 \mathrm{nM}$ retinoid antagonist AGN194310 for the indicated times, and the cell lysates were processed for immunoblot determination of phosphorylated extracellular signal regulated-kinases (pERK1/2), p-p38 and phosphorylated c-Jun N-terminal kinase ( $p J N K$ ) mitogen-activated protein kinase (MAPK) (A), and phosphorylated activating transcription factor 2 ( $p$ ATF2) (B).C: The level of activated p38 MAPK activity was determined by a kinase activity assay. ATDC5 cells were treated with $20 \mu M$ ERK1/2 kinase inhibitor PD98059, p38 MAPK SB203580 or JNK inhibitor SP60012 and then stimulated with $100 \mathrm{nM}$ AGN204647 for 24 h. Total RNA from these cells was used for a real-time reverse transcription-polymerase chain reaction analysis for Agr (D), Col2 (E), CollOA1 (F), Tg2 (G), Mmp13 (H), and Ccn2 (I). The values in the graph indicate the relative $m R N A$ level. Two independent experiments were performed, and the average of the mRNA level is shown. 
the levels of phosphorylated versus total levels of the three kinases. As shown in Figure 3A, expression of ERK1/2, p38, and JNK-activated MAPK started to increase after the RAR $\gamma$ agonist treatment. ERK1/2 and JNK MAPK activation reached a plateau at $30 \mathrm{~min}$, whereas p38 MAPK activation reached a maximum at $24 \mathrm{~h}$. Retinoid antagonist at $100 \mathrm{nM}$ completely inhibited the p38 MAPK activation for $24 \mathrm{~h}$, and weakly suppressed pERK1/2 for $60 \mathrm{~min}$. However, pJNK was not changed by the treatment with retinoid antagonist.

To clarify the phosphorylation of ATF2 through RAR $\gamma$ signaling, cells were treated with retinoid antagonist and RAR $\gamma$ agonist. The treatment of cells with retinoid antagonist blocked RAR $\gamma$-induced ATF2 phosphorylation (Figure 3B). We further confirmed the activation of ATF2 by conducting a p38 MAPK activity assay. p38 MAPK activity was up-regulated by treatment with $\operatorname{RAR} \gamma$ agonist and inhibited by retinoid antagonist (Figure 3C).

To determine whether MAPKs were involved in RAR $\gamma$ action on the mRNA expression of aggrecan, Col2, Col10A1, Tg2, Mmp13 and Ccn2, ATDC5 cells were treated with $\operatorname{RAR} \gamma$ agonist for $24 \mathrm{~h}$ with/without specific inhibitors of MAPK/ERK kinase (MEK), p38 MAPK, or p-JNK. The aggrecan and Col2 mRNA levels were not significantly changed by the treatment with any of the inhibitors (Figure 3D and E). The treatment with p38 MAPK inhibitor reversed RAR $\gamma$ agonist-induced mRNA expressions of Col2, Col10A1, Tg2, Mmp13 and Ccn2. However, MEK inhibitor did not affect the RAR $\gamma$ agonist-induced Col10A1, Mmp13 and $C c n 2$ expression (Figure $3 \mathrm{~F}, \mathrm{H}$ and $\mathrm{I}$ ).

\section{Discussion}

The importance of RAR in chondrocyte maturation and function was demonstrated in in vitro studies $(11,14)$ and has been re-affirmed by a recent analysis of mice deficient in $\operatorname{RAR} \alpha / \operatorname{RAR} \gamma$ or $\operatorname{RAR} \beta / \operatorname{RAR} \gamma$, that exhibited significant skeletal growth retardation (10). Our results here show that Rarg was specifically expressed in proliferative and prehypertrophic zones of mouse tibial long bone growth plates, with CollOA1, Mmp13 and Ccn2 being the most abundantly expressed members and their expressions prominently characterizing hypertrophic chondroyctes.

It was recently reported that a general deletion of Rarg but not Rara in mice caused bone marrow defects characterized by hematopoietic stem cell alteration and even a marked reduction in trabecular bone during aging $(15,16)$. It is, thus, clear that there are intimate cross-talk interactions between the hypertrophic growth plate and bone marrow that are essential for the physiological progression of skeletal growth. Our present data indicate that RAR $\gamma$ signaling may bring about appropriate expression levels and the actions of these important hypertrophic chondrocyte-specific downstream effectors, contributing to a seamless transition from hypertrophic cartilage to trabecular bone. The Rarg gene expression is consistent with the gene expressions of Mmp13 and Ccn2, which have important roles in the resorption of hypertrophic cartilage from growth plates and the remodeling of newly deposited trabecular bone during long bone development $(2,4-6)$.

Our evidence obtained from ATDC5 cell cultures shows that an exogenous RAR $\gamma$ agonist stimulated Mmp13 and Ccn2 expression, and a retinoid antagonist reversed this action. Based on these and other observations, it is clear that retinoid signaling is an important regulator of the expression of Mmp13 and Ccn2 in chondrocytes, and that Mmp13 and Ccn2 may be important downstream effectors. The three major MAPK signaling pathways are involved in the program of chondrogenic differentiation from embryonic through postnatal stages of development (17). The murine ATDC5 cell line has been used as monolayer culture to identify the MAPK transducers downstream of the chondrostimulatory growth factors (18). Insulin also induced the phosphorylation of all three MAPK pathways in ADTC5 cells (19). Indeed, the p38 MAPK pathway regulates the expression of CoI2 and aggrecan and proteoglycan synthesis in response to growth factors $(18,20,21)$.

In our analyses using ATDC5 cells, it was difficult to evaluate the effect of the MAPK inhibitor on the ColII and aggrecan mRNA expressions. Notably, p38 MAPK signaling has been shown to positively regulate hypertrophic chondrocyte differentiation with an up-regulation of CollOAl, and Mmp13 (22). In a related study, Zhang et al. (23) found that the expression in transgenic mouse chondrocytes of a constitutively active mutant of Mmk6 (a MAPK kinase that specifically activates p38) had a dwarf phenotype characterized by reduced chondrocyte proliferation, the inhibition of hypertrophic differentiation, and a delay in the formation and secondary ossification centers. These studies reached the conclusion that activated p38 MAPK has a positive role in chondrocyte maturation, hypertrophy, and the remodeling of newly-deposited trabecular bone during long bone development.

Retinoids inhibit cell proliferation by suppressing the phosphorylation of ERK1/2 (24). In contrast, p-p38 stimulated genes Rarg and ColloAl, whereas p-ERK stimulated the expression of genes including $C c n 2$ and osteopontin in primary cultured chick hypertrophic chondrocytes by ATRA (8). In contrast to our finding, p38 MAPK reduced RAR $\gamma$ signal-induced $C c n 2$ expression in ATDC5 cells. We observed that treatment with the JNK inhibitor SP60012 had no impact on the chondrocyte differentiation.

All these studies point to an involvement of p38 pathway signaling in the regulation of chondrocyte differentiation hypertrophy. However, further studies are needed to clarify the specific roles of $\operatorname{RAR} \gamma$ signaling on the $\mathrm{p} 38$ cascade in endochondral bone formation. 


\section{Conflicts of Interest}

None of the Authors have any conflict of interest in regard to this study.

\section{Acknowledgements}

This work was partly supported by a Grant-in-Aid for Scientific Research (B) \#JP18H02999 (T.S.) from Japan Society for the promotion of Sciences, Japan and the NIH AR05683 (M.I.) and NIH AR072713 (M.I.).

\section{References}

1 Lui JC, Nilsson O and Baron J: Recent research on the growth plate: Recent insights into the regulation of the growth plate. $\mathrm{J}$ Mol Endocrinol 53(1): T1-9, 2014.

2 Ivkovic S, Yoon BS, Popoff SN, Safadi FF, Libuda DE, Stephenson RC, Daluiski A and Lyons KM: Connective tissue growth factor coordinates chondrogenesis and angiogenesis during skeletal development. Development 130(12): 2779-2791, 2003.

3 Kubota S and Takigawa M: Role of CCN2/CTGF/HCS24 in bone growth. Int Rev Cytol 257: 1-41, 2007.

4 Vu TH, Shipley JM, Bergers G, Berger JE, Helms JA, Hanahan D, Shapiro SD, Senior RM and Werb Z: MMP-9/gelatinase B is a key regulator of growth plate angiogenesis and apoptosis of hypertrophic chondrocytes. Cell 93(3): 411-422, 1998.

5 Inada M, Wang Y, Byrne MH, Rahman MU, Miyaura C, LopezOtin C and Krane SM: Critical roles for collagenase-3 (Mmp13) in development of growth plate cartilage and in endochondral ossification. Proc Natl Acad Sci USA 101(49): 17192-17197, 2004.

6 Stickens D, Behonick DJ, Ortega N, Heyer B, Hartenstein B, Yu Y, Fosang AJ, Schorpp-Kistner M, Angel P and Werb Z: Altered endochondral bone development in matrix metalloproteinase 13deficient mice. Development 131(23): 5883-5895, 2004.

7 Ortega N, Behonick DJ, Colnot C, Cooper DN and Werb Z: Galectin-3 is a downstream regulator of matrix metalloproteinase- 9 function during endochondral bone formation. Mol Biol Cell 16(6): 3028-3039, 2005.

8 Shimo T, Koyama E, Sugito H, Wu C, Shimo S and Pacifici M: Retinoid signaling regulates CTGF expression in hypertrophic chondrocytes with differential involvement of MAP kinases. J Bone Miner Res 20(5): 867-877, 2005.

9 Pacifici M: Retinoid roles and action in skeletal development and growth provide the rationale for an ongoing heterotopic ossification prevention trial. Bone 109: 267-275, 2018

10 Williams JA, Kondo N, Okabe T, Takeshita N, Pilchak DM, Koyama E, Ochiai T, Jensen D, Chu ML, Kane MA, Napoli JL, Enomoto-Iwamoto M, Ghyselinck N, Chambon P, Pacifici M and Iwamoto $\mathrm{M}$ : Retinoic acid receptors are required for skeletal growth, matrix homeostasis and growth plate function in postnatal mouse. Dev Biol 328(2): 315-327, 2009.

11 Koyama E, Golden EB, Kirsch T, Adams SL, Chandraratna RA, Michaille JJ and Pacifici M: Retinoid signaling is required for chondrocyte maturation and endochondral bone formation during limb skeletogenesis. Dev Biol 208(2): 375-391, 1999.

12 Koyama E, Young B, Nagayama M, Shibukawa Y, EnomotoIwamoto M, Iwamoto M, Maeda Y, Lanske B, Song B, Serra R and Pacifici M: Conditional KIF3A ablation causes abnormal hedgehog signaling topography, growth plate dysfunction, and excessive bone and cartilage formation during mouse skeletogenesis. Dev 134(11): 2159-2169, 2007.

13 Shimono K, Tung WE, Macolino C, Chi AH, Didizian JH, Mundy C, Chandraratna RA, Mishina Y, Enomoto-Iwamoto M, Pacifici $\mathrm{M}$ and Iwamoto $\mathrm{M}$ : Potent inhibition of heterotopic ossification by nuclear retinoic acid receptor- $\gamma$ agonists. Nat Med 17(4): 454-460, 2011.

14 Iwamoto M, Shapiro IM, Yagami K, Boskey AL, Leboy PS, Adams SL and Pacifici M: Retinoic acid induces rapid mineralization and expression of mineralization-related genes in chondrocytes. Exp Cell Res 207(2): 413-420, 1993.

15 Purton LE, Dworkin S, Olsen GH, Walkley CR, Fabb SA, Collins SJ and Chambon P: RARgamma is critical for maintaining a balance between hematopoietic stem cell self-renewal and differentiation. J Exp Med 203(5): 1283-1293, 2006.

16 Walkley CR, Olsen GH, Dworkin S, Fabb SA, Swann J, McArthur GA, Westmoreland SV, Chambon P, Scadden DT and Purton LE: A microenvironment-induced myeloproliferative syndrome caused by retinoic acid receptor gamma deficiency. Cell 129(6): 1097-1110, 2007.

17 Bobick BE and Kulyk WM: Regulation of cartilage formation and maturation by mitogen-activated protein kinase signaling. Birth Defects Res C Embryo Today 84(2): 131-154, 2008.

18 Nakamura K, Shirai T, Morishita S, Uchida S, Saeki-Miura K and Makishima F: p38 mitogen-activated protein kinase functionally contributes to chondrogenesis induced by growth/differentiation factor-5 in ATDC5 cells. Exp Cell Res 250(2): 351-363, 1999.

19 Nakajima M, Negishi Y, Tanaka H and Kawashima K: p21(Cip1/SDI-1/WAF-1) expression via the mitogen-activated protein kinase signaling pathway in insulin-induced chondrogenic differentiation of ATDC5 cells. Biochem Biophys Res Commun 320(4): 1069-1075, 2004.

20 Yosimichi G, Nakanishi T, Nishida T, Hattori T, TakanoYamamoto T, Takigawa M: CTGF/Hcs24 induces chondrocyte differentiation through a p38 mitogen-activated protein kinase (p38MAPK), and proliferation through a p44/42 MAPK/extracellular-signal regulated kinase (ERK). Eur J Biochem 268(23): 6058-6065, 2001.

21 Tuli R, Tuli S, Nandi S, Huang X, Manner PA, Hozack WJ, Danielson KG, Hall DJ and Tuan RS: Transforming growth factor-beta-mediated chondrogenesis of human mesenchymal progenitor cells involves $\mathrm{N}$-cadherin and mitogen-activated protein kinase and Wnt signaling cross-talk. J Biol Chem 278(42): 41227-41236, 2003.

22 Stanton LA, Sabari S, Sampaio AV, Underhill TM and Beier F: p38 MAP kinase signalling is required for hypertrophic chondrocyte differentiation. Biochem J 378(Pt 1): 53-62, 2004.

23 Zhang R, Murakami S, Coustry F, Wang Y and de Crombrugghe B: Constitutive activation of MKK6 in chondrocytes of transgenic mice inhibits proliferation and delays endochondral bone formation. Proc Natl Acad Sci USA 103(2): 365-370, 2006.

24 Dalvin S, Komatsuzaki K, Anselmo MA, Kling DE, Schnitzer JJ and Kinane TB: Retinoic acid decreases fetal lung mesenchymal cell proliferation in vivo and in vitro. Dev Growth Differ 46(3): 275-282, 2004.

Received October 16, 2018

Revised November 20, 2018 Accepted November 21, 2018 